\title{
Review of Gait, Cognition, and Fall Risks with Implications for Fall Prevention in Older Adults with Dementia
}

\author{
Weihong Zhanga, b Lee-Fay Low ${ }^{a} \quad$ Michael Schwenk $^{c} \quad$ Nicholas Mills $^{d, e}$ \\ Josephine Diana Gwynn ${ }^{a}$ Lindy Clemson ${ }^{a}$ \\ ${ }^{a}$ Faculty of Health Sciences, University of Sydney, Sydney, NSW, Australia; ${ }^{b}$ Department of \\ Occupational Therapy, Wolper Jewish Hospital, Sydney, NSW, Australia; ' ${ }^{\mathrm{N}}$ Network Aging \\ Research, Heidelberg University, Heidelberg, Germany; ${ }^{d}$ Department of Geriatric Medicine, \\ St Vincent's Hospital, Sydney, NSW, Australia; ${ }^{e}$ Department of Medicine, University of New \\ South Wales, Sydney, NSW, Australia
}

\section{Keywords}

Cognitive impairment · Executive function · Risk factors - Theoretical framework · Falls prevention

\begin{abstract}
Background: Older people with cognitive impairment are at increased risk of falls; however, fall prevention strategies have limited success in this population. The aim of this paper is to review the literature to inform a theoretical framework for fall prevention in older adults with dementia. Summary: A narrative review was conducted on fall risk factors in people with cognitive impairment, the relationship between cognition and gait, and their joint impact on the risk of falls. This was used to develop a theoretical framework for fall prevention for people with dementia. Executive function and motor function are closely related as they share neuroanatomy. This close relationship has been confirmed by observational studies including neuroimaging and intervention studies. Executive function is the cognitive domain most commonly associated with gait dysfunction. Attention, sensory integration, and motor planning are the sub-domains of executive function associated with risk of falls through gait dysfunction, whereas cognitive flexibility, judgement, and inhibitory control affect risk of falls through risk-taking behaviour. Key Messages: Gait, cognition, and falls are closely related. The comorbidity and interaction between gait abnormality and cognitive impairment may underpin the high prevalence of falls in older adults with dementia. Gait assessment and cognitive assessment, particularly executive function, should be integrated in fall risk screening. Assessment results should be interpreted and utilised using a multidisciplinary approach; specific strategies such as customised gait training and behavioural modulation should be considered as part of falls prevention for people with dementia.


Dementia

and Geriatric

Cognitive Disorders

\begin{tabular}{l|l}
\hline Dement Geriatr Cogn Disord 2019;48:17-29 \\
\hline DOI: 10.1159/000504340 & $\begin{array}{l}\text { c 2019 S. Karger AG, Basel } \\
\text { www.karger.com/dem }\end{array}$ \\
\hline
\end{tabular}

Zhang et al.: Review of Gait, Cognition, and Fall Risks

\section{Introduction}

Falls are a major cause of injury to older people, and risk of falls increases when older people are cognitively impaired [1]. The rate of falls in community-dwelling older people with dementia is twice that of a cognitively intact population with almost two thirds of people with dementia falling annually [2]. The theories underlying efficacious fall prevention interventions for older adults are well documented [3]. However, fall prevention strategies that are successful in cognitively intact older adults have limited success in reducing fall risk in people with cognitive impairment. Consequently, prominent fall prevention guidelines do not provide specific recommendations for people with cognitive impairment due to insufficient evidence [4].

The aim of this paper is to review the literature to inform a theoretical framework for fall prevention programs in older adults with dementia. Based on current findings from systematic reviews, clinical studies, and population-based studies, the paper draws together results from across a broad range of research, including risk factors for falls, the relationship between cognition and gait, and the joint impact of cognition and gait on falls.

\section{Risk Factors for Falls}

Cognitively intact and cognitively impaired older adults share some well-established risk factors for falls, such as fall history, environmental hazards, motor impairments, and visual impairments $[5,6]$. However, there are other fall risk factors that are more specific to people living with dementia.

We categorise the factors relating to falls in this review as medical, environmental, physical and sensory, cognitive, and psychological factors (Fig. 1). The underlined factors in Figure 1 are specifically relevant to older adults with dementia.

\section{Medical Factors}

Orthostatic hypotension (a common cause of syncope) and medication side effects have special features in older people with dementia. Syncope is the primary cause of hospitalisation in elderly patients with dementia [7], and drug-induced orthostatic hypotension is its most frequent cause; however, it is often underdiagnosed due to these people's inability to verbalise or remember specific complaints and to cooperate with laboratory work-up [8]. Psychotropic medications including drugs commonly used to manage behavioural and psychological symptoms of dementia (e.g., antipsychotics, benzodiazepines) increase the risk of falls $[5,9]$. The use of acetylcholinesterase inhibitors (AChEIs) and N-methyl-D-aspartate (NMDA) receptor antagonists to treat dementia symptoms has been reported to present a $63 \%$ increased hazard of falling over time, which may result from the increased risk of syncope [10].

\section{Physical and Sensory Factors}

People with dementia are more sedentary and perform less physical activity than cognitively healthy controls [11]. Sedentary behaviour is strongly related to negative health outcomes and mortality, and is associated with lower cognitive performance [11]. A decline in executive function can lead to apathy and lack of motivation of goal-directed behaviour [12]. These individuals may lack the interest or motivation to engage in activities of daily living, in which strength and balance are basic requirements. This decline in physical functioning may lead to increased risk of falls. 
Fig. 1. Risk factors for falls in older adults with dementia (the underlined factors are those specifically relevant to older adults with dementia).

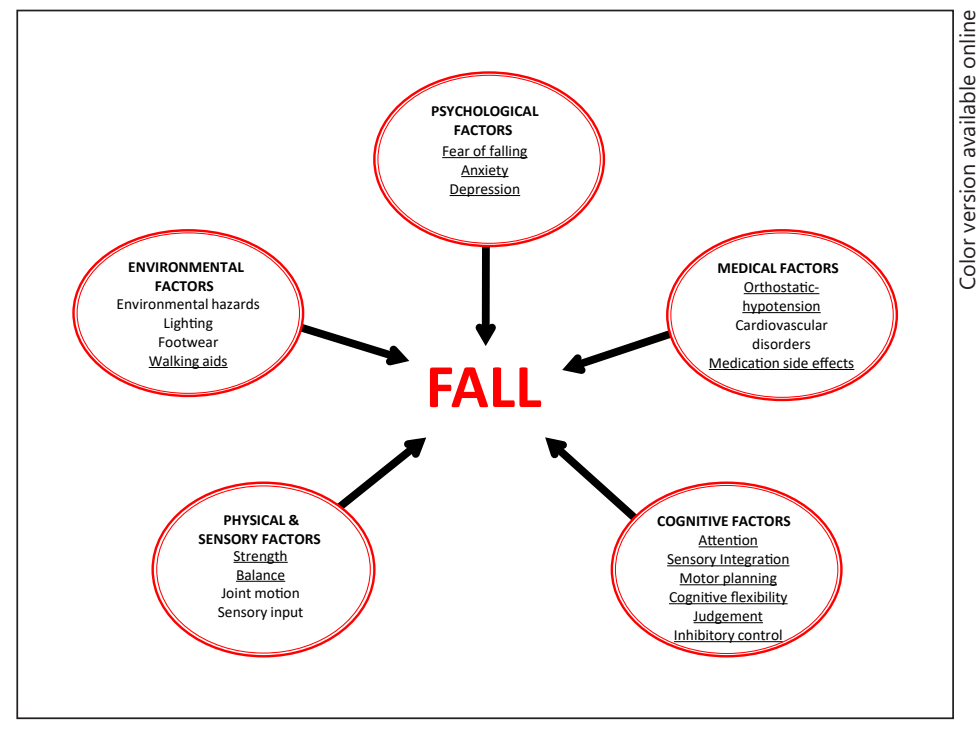

\section{Psychological Factors}

Psychological factors such as depression, anxiety, impulsivity, and behavioural disturbances are associated with increased fall risk in older adults with dementia [4]. Wandering may increase the fall risks because more time is spent in standing and walking, and this risk may be exacerbated if wandering behaviour is combined with impulsivity, agitation, and poor balance control [9]. A cohort study demonstrated that longer standing and short walking bouts (an indication of restlessness and agitation) increase the risk of falls in people with dementia [13]. Anxiety may relate to the underlying pathology and neuro-chemical changes associated with dementia, and may increase fall risk by impeding postural responses to perturbations [9]. Fear of falling, which is associated with anxiety [9], is more common in older adults with mild cognitive impairment or early-stage dementia, and may increase fall risks in this population [14].

\section{Environmental Factors}

Ambulation with a walking aid is a real-life situation of multitasking (walking and manoeuvring a walking aid), which requires high levels of motor control and cognitive response to multiple sensory inputs and environmental conditions [15]. The attentional demands associated with the use of a walking aid could lead to an impaired ability to maintain or recover balance in older people [16]. Walking aids may be prescribed to facilitate independent mobility; however, if the person lacks the cognitive capacity, ambulating with a walking aid may lead to instability, unsafe use of the aid, and falls [15].

According to the most recent systematic review and meta-analysis on risk factors for falls in community-dwelling older people, gait problems and cognitive impairment are among those factors highly associated with risk of falling [17]. The impact of cognition and gait on falls will be discussed in detail below.

\section{The Relationship between Cognition and Gait}

\section{Cognition}

The Diagnostic and Statistical Manual of Mental Disorders (DSM-5) categorised six principal domains of cognitive function - complex attention, executive function, learning and 


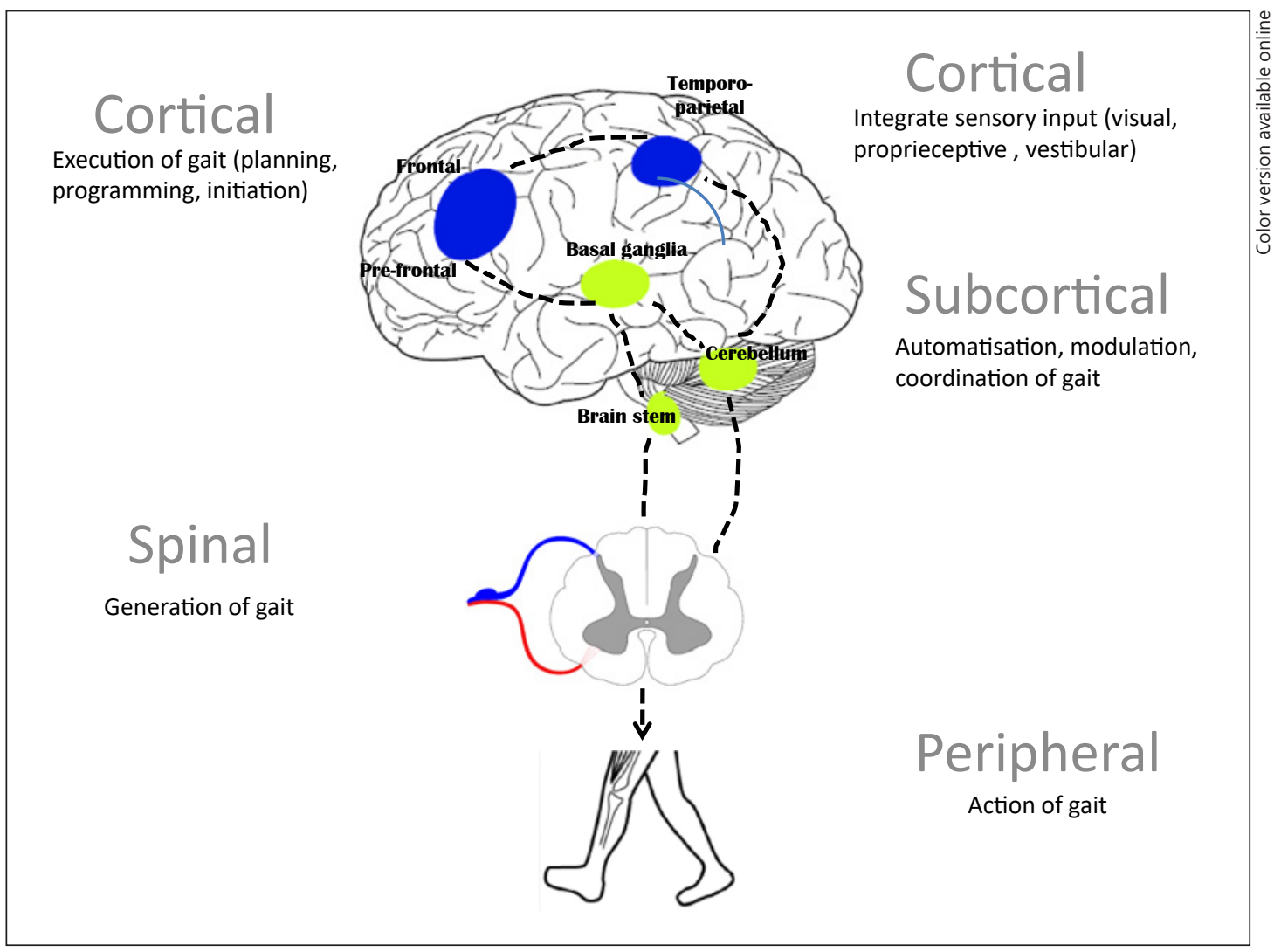

Fig. 2. Neural pathways in motor control.

memory, language, perceptual-motor function, and social cognition - each with sub-domains [18]. Complex attention (sustained attention, divided attention, selective attention, and processing speed), executive function (planning, decision making, working memory, responding to feedback, inhibition, and flexibility), and perceptual-motor function (visual perception, visuoconstructional reasoning, perceptual-motor coordination) [18] are most relevant to fall risks.

Gait

Gait is the pattern of walking and is an attention-demanding, high-level, controlled task [19]. Normal gait requires an ability to integrate sensory input (visual, proprioceptive, and vestibular), motor planning and execution (planning, initiation, automatisation, integration, and coordination of gait), as well as an intact musculoskeletal system and supporting cardiovascular system [20]. Gait control involves all levels of the neural pathway, namely cortical, subcortical, spinal, and peripheral (Fig. 2). Sensory integration, motor planning, and execution of gait occur at the cortical-subcortical level. Basal ganglia, cerebellum, and cerebral cortex are involved in the planning and execution of purposeful locomotion [21].

Gait disorders are frequent in older adults and lead to falls, immobility, and increased mortality [22]. The cause of gait disorders can be classified as either neurological or nonneurological, depending on whether the neural pathways have been affected [23]. A clinical gait classification divides gait abnormalities into low-, middle-, and high-level disorders [24, 25]. The lower levels include peripheral musculoskeletal and sensory disorders. Middle-level disorders include hemiplegic, cerebellar, and dystonic gaits in which the neurological deficits 
found are consistent with the gait disturbance [25]. Higher-level gait disorders, such as cautious gait and frontal gait disorders, are due to disturbances of the highest sensorimotor systems that cannot be accounted for by the neurological signs [25], and are thought to result from disruptions in cortico-cortical and cortico-subcortical connections [26]. The higherlevel disorder is the category that dementia-related gait disorders belong to.

\section{Cognition and Gait Are Closely Related}

Clinical and research evidence has established a close relationship between gait and cognition. Cognitive impairment co-exists with gait decline even in the early stages of neurodegenerative diseases [27]. People with cognitive impairment display changes in gait parameters [28], and changes in gait can be used as an early biomarker of dementia [29, 30]. Recent evidence has demonstrated that cognitive impairment and falls are related; gait impairments and falls are more prevalent in people with dementia, and the prevalence of gait impairments and falls increases with the severity of cognitive impairment [31]. The close relationship between gait and cognition, and their joint impact on falls may be the underlying mechanism explaining the increased fall risks in older adults with dementia.

\section{Shared Anatomy and Pathology between Cognition and Gait}

Evidence from Imaging Studies

Motor and cognitive functions share genetic determinants, and cognitive and gait control share neural networks [32]. Gait control is predominately mediated by frontal subcortical circuits, which overlap with circuits controlling executive function [23]. Within the five major frontal subcortical circuits, two of which are related to motor control and the other three are involved in controlling executive function in the aspects of planning, working memory, rulebased learning, attention, and emotional regulation [33].

Gait and cognitive disorders also share common pathology, such as vascular changes and neurodegeneration [23]. Accumulation of vascular structural abnormalities, in both white and grey matter, accounts for cognitive decline and gait impairment [34]. The periventricular white matter sub-serves gait and balance control, and controls cognitive processing speed and executive function. White matter lesions may affect motor and cognitive functions simultaneously due to the close proximity of these two circuits [23].

Imaging studies have confirmed the shared anatomic and pathological basis for both cognitive and motor function. These studies use magnetic resonance imaging (MRI) to evaluate brain volume or tissue integrity and their link to gait and cognitive function. It has been demonstrated that reduced volumes in prefrontal and frontoparietal regions are associated with impaired gait variables [35, 36]; white-matter hyperintensities are associated with gait disturbances and cognitive impairment [36]. A review on imaging studies suggested that widespread grey matter atrophy in areas associated with cognition, such as the frontal lobe and parietal lobe, was also associated with gait dysfunction [37].

Functional near-infrared spectroscopy (fNIRS) is a neuroimaging technique that measures changes in cortical brain oxygenated haemoglobin $\left(\mathrm{HbO}_{2}\right)$ levels [38]. fNIRS studies revealed differential involvement of prefrontal, premotor, and motor cortices in gait tasks [21]. Significant increase in $\mathrm{HbO}_{2}$ levels was found in walking-while-talking compared to normal walking in older adults [38]; cortical activation had increased in the prefrontal cortex during a postural control task for individuals with Parkinsonian syndromes [39]. The involvement of frontal and prefrontal circuits in cognitively demanding locomotion tasks affirms cognitive processes in mobility [21].

Evidence from Observational Studies

Many observational studies have explored the relationship between specific cognitive domains and gait abnormalities. A review [19] revealed the importance of executive function 
and attention to the performance of gait during normal walking, as well as in ageing and in pathological conditions. In a population-based study of community-dwelling older people, executive function/attention and processing speed were associated with performance on most absolute gait measures (e.g., gait speed, step length, double support phase) [40]. Another study also indicated that poor performance in executive function was associated with increased gait variability, especially during tasks that demanded cognitive skills in older adults [36].

Evidence from Interventional Studies

Interventional studies have confirmed the close relationship between gait disorders and cognitive impairment. Physical activities and exercises appear to enhance the functioning of the prefrontal brain region as well as visuospatial performance [41]. Older adults with higher aerobic fitness levels may have a greater brain volume, the so-called exercise-induced brain changes. They are able to recruit additional brain resources to improve performance on various cognitive and motor tasks than their sedentary counterparts [41]. Aerobic training appears to trigger global neuroplastic effects and shows increased efficiency in brain regions associated with executive control processes [42]. It has robust but selective benefits for cognition - the greatest benefits occur for executive functions, which indicates that exercise may help reduce falls by improved executive functions [43].

A recent review explored the relationship between gait and cognition in ageing and neurodegeneration and highlighted the role of brain motor control deficits in fall risks [31]. It concluded that both pharmacological and non-pharmacological cognitive interventions may improve gait performance and reduce fall risks in older adults with cognitive impairment [31]. Non-pharmacological cognitive interventions (cognitive training, dual-task training, and virtual reality modalities) may improve gait performance, especially during dual-task walking [31]. Cognitive remediation improves gait velocity and dual-task gait velocity; visually enhanced treadmill training improves walking adaptability and fear of falling in older adults with executive dysfunction [44].

Cognitive enhancers may improve gait variability in people with Alzheimer's disease (AD) [45]. AChEIs (i.e., donepezil, galantamine, and rivastigmine) and NMDA receptor antagonists (i.e., memantine) are symptomatic drugs for the treatment of AD [46]. Because higher levels (subcortical and cortical levels) of gait control are closely linked to the cerebral neural network's integrity and efficiency, it has been suggested that supplementing the cholinergic loss with AChEIs and preventing the loss of glutamatergic neurons with memantine may improve cognition and, in consequence, gait performance [47]. The role of drugs designed to enhance attention and executive function as a therapeutic option for reducing fall risk has been supported by recent findings [31].

\section{Joint Impact of Cognition and Gait on Risk of Falls}

Normal walking consists of three primary components: locomotion, including initiation and maintenance of rhythmic stepping, balance, and ability to adapt to the environment [48]. Furthermore, walking requires strategic planning of the best route, continuous interaction with the environment and internal factors [48], as well as adaptation of the movement to the incoming sensory information [49]. While walking itself might be a routine process, the incoming sensory information and its use to adapt the movement requires executive function processes. A review [50] indicated that loss in specific cognitive domains, such as executive function, attention, information processing, and reaction time, were consistently associated with an increased fall risk. 


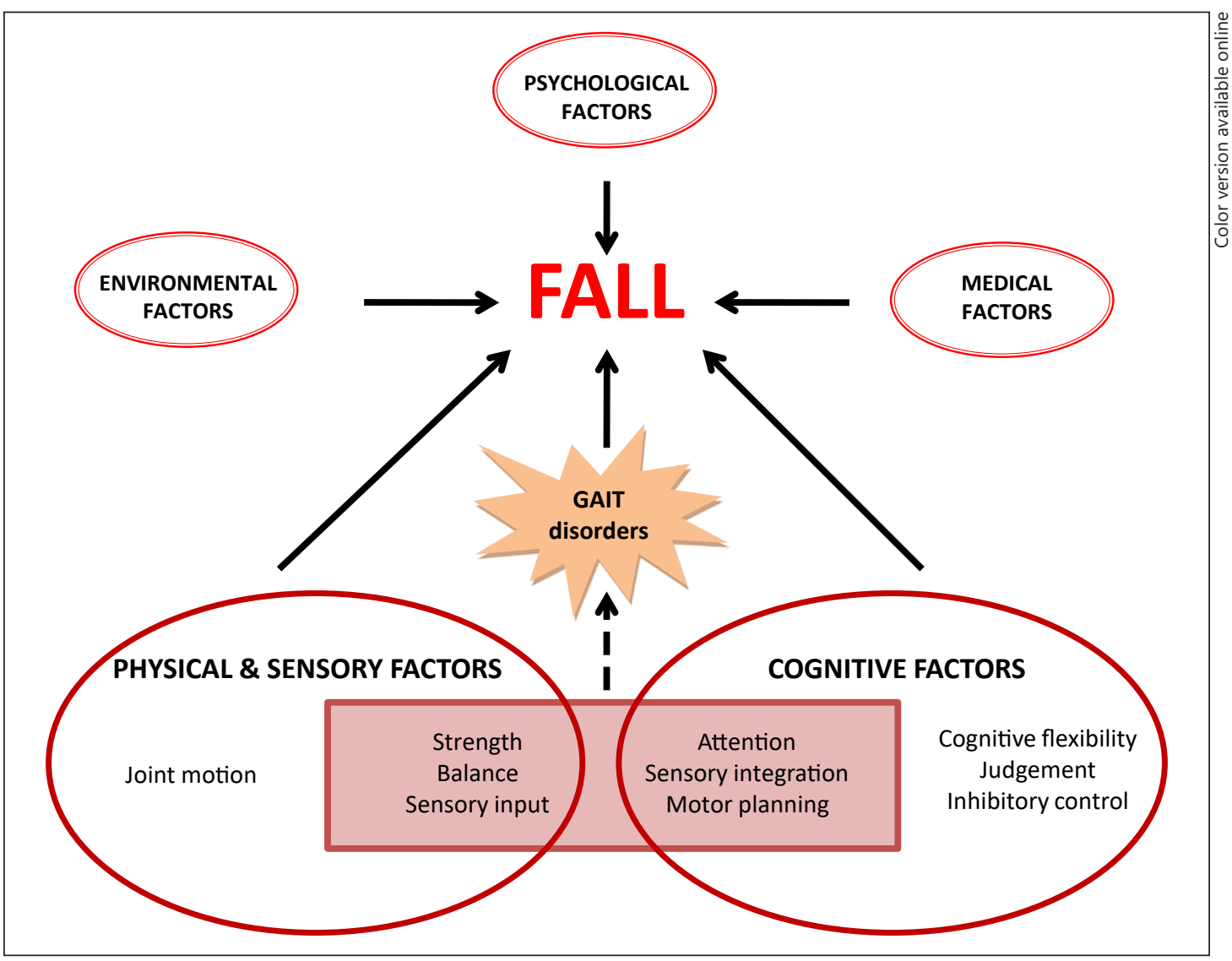

Fig. 3. Relationship between gait and falls in older adults with dementia.

Executive function is a set of higher-order cognitive processes that control, integrate, organise, and maintain other cognitive abilities [51]. Task planning, sensory integration, judgment, problem solving, and reasoning are components of the executive function that are intuitively related to safe navigation and mobility in complex everyday environments [52]. Executive function plays a role to compensate for age-associated decline in motor function and to allow for fall-free gait in complex, everyday situations [53]. It is the domain most commonly associated with gait dysfunction [28]. The sub-domains of attention, sensory integration, and motor planning within executive function are most related to gait dysfunction, and thus impact on fall risk (Fig. 3).

Figure 3 illustrates the relationship between gait and falls in older adults with dementia. Strength, balance, and sensory input within the physical and sensory factors, together with attention, sensory integration, and motor planning within the cognitive factors (the factors inside the box), have a direct impact on gait performance, and thus result in increased risk of falls in this population. Other factors within the physical and sensory and cognitive factors, such as inhibitory control and judgement, may also contribute to the risk of falls; however, their impact is not mediated by gait.

\section{Gait-Associated Cognitive Factors for Fall Risks}

Attention

Attention is a specific type of executive function. It is a dynamic function driven by sensory perception and the need to select a preferred stimulus for a particular action while ignoring 
the unnecessary and the irrelevant [52]. Selective attention is focused on sensory input - any changes to sensory input, such as reduced visual acuity, may affect gait and might need executive function and attention to play a larger role to maintain stability [54]. A study using functional MRI indicated that older adults show greater cognitive monitoring of movement than younger adults, and this was thought to be related to sensory decline [54]. Divided attention, which refers to the ability to carry out more than one task at the same time, has been found to be most closely related to balance, gait, and fall risk [50]. Impairment in executive function and attention may prevent fallers from devoting the appropriate attentional resources to their balance and gait, reduce their ability to confront and adapt to challenging environments (e.g., obstacles, uneven path), and may lead to an increased fall risk [55]. Dual-tasking cost (the difference between single-task and dual-task performance) measures the effect of divided attention deficits in $\mathrm{AD}$, and also supports the hypothesis that attention is an important component in the control of walking [54].

Processing speed is one component of complex attention [18]. Many cognitive operations require sufficiently quick information processing speed for relevant operations to be executed within the time allowed; slowed processing speed often underlies attentional deficits [56]. Visual attention and visual processing speed in older adults are independently associated with mobility problems [54], which becomes more important in gait control when other sensory input (i.e., vestibular, proprioception) declines. Cautious gait may be a likely outcome when sensory input declines and processing speed decreases [54].

Sensory Integration

Balance, or postural stability, is a complex process that involves the integration of information from the musculoskeletal systems and the sensory input (vision, proprioception, and the vestibular system), in the presence of cognition [57]. The motor and sensory systems are linked by higher-order cortical processes, which are required for planning movements, solving problems, divided attention, and responding to changes within the environment [50]. People with dementia may have impaired inter-hemispheric transfer of information; this cortico-cortical disconnection, as seen in AD patients, may disrupt effective interaction between different processing areas [58]. The widespread involvement of white matter in mobility among older adults from imaging studies suggests that locomotion is dependent on the integrity and communication of multiple tracks across both hemispheres [21]. Imaging studies investigating the associations between neuropathology and gait indicate a strong relationship between the parietal lobe and gait as parietal regions are central to sensory integration, visuospatial function, and managing the relationship between one's self and surroundings [37]. Therefore, people with dementia may have impaired ability to integrate sensory information to facilitate performance in postural stability and gait.

\section{Motor Planning}

Walking in a real-life situation is a goal-directed gait task, especially when turning, avoiding obstacles, and passing through narrow spaces. Execution of these goal-directed movements requires complex conscious control including efficient evaluation of the spatial properties and efficient planning [59]. This "modulated gait" is controlled through a specific pathway involving prefrontal cortex projections through the subthalamic nucleus and downstream to the locomotor centres of the brainstem, and this is only employed during gait tasks that require a higher level of processing [60]. The parieto-frontal circuits subserve visuomotor functions for motor planning and movement reprogramming [59]. Deficits in motor planning and programming may cause gait dysfunction. 


\section{Non-Gait-Associated Cognitive Factors for Fall Risks}

Other executive function sub-domains - cognitive flexibility, judgement, and inhibitory control - also impact on fall risks in older adults with dementia (Fig. 3). However, they may not cause gait dysfunction, but rather directly impact on fall risks.

Cognitive Flexibility

Cognitive flexibility is a sub-domain of executive function and is required for adapting behaviour to external influence [61]. The ability to adapt to changing contingencies is a key element that is associated with an increased risk of falls [62]. Adaptive walking requires integration of the information from both the body and the environment, and frequent alterations in the coordination of stepping patterns and trunk motion [63]. Individuals with poor cognitive flexibility reach the maximum adaption capability of their walking pattern earlier than those with good cognitive flexibility [61]. Lack of cognitive flexibility to adapt and prioritise gait in circumstances of challenge, such as obstacles or changes in surface and lighting, may result in falls [62].

\section{Judgement}

Although individuals with dementia may appear to walk slowly, they may be walking faster than their motor and cognitive abilities safely allow, leading to a greater risk of falling [31]. This could be explained by their reduced ability to appraise the hazards in the environment [31], or their lack of insight which results in the inability to adapt their gait speed to reflect their physical or cognitive impairments [6]. Impaired executive function may also increase fall risks by altering older adults' judgment during motor planning for daily activities, such as reaching [64]. People with dementia may misjudge possible dangers in their environment. They may overestimate their own capabilities concerning mobility, which may cause falls [6].

\section{Inhibitory Control}

Inhibitory control involves being able to control one's attention, behaviour, thoughts, and/or emotions to override a strong internal predisposition or external lure, and instead do what is more appropriate or needed [65]. It is an important aspect of cognitive function related to fall risks as it creates a selective focus on information or an action to the exclusion of other sensory inputs and motor outputs [66]. Without inhibitory control, one's action would be driven by impulses, old habits of thought, and/or stimuli in the environment [65] and may cause failure to accurately appraise the risk of activities that were once safe [31], and as a result falls may occur.

Figure 3 demonstrates how cognition, gait, and falls are linked and how cognitive factors impact on falls, either via gait disorders or directly lead to falls through risk-taking behaviour due to cognitive impairment.

\section{Implications and Conclusion}

Gait, cognition, and fall risks are areas of increasing research interest. The literature suggests that there is a relationship between gait and cognition, gait abnormality and cognitive impairment, and fall risks and cognitive impairment. Previous reviews have explored the dual-task paradigm and demonstrated the cognitive contributions to gait and falls [31, 36, 42] and revealed risk factors for falls among older adults [49]. This paper has brought together the three concepts - gait, cognition, and fall risks with a particular focus on older adults with dementia, and has provided a detailed explanation on dementia-related fall risk factors and 
Zhang et al.: Review of Gait, Cognition, and Fall Risks

the mechanism of the impact of individual cognitive factors. Being dementia specific with in-depth analysis has enabled this theoretical framework to provide clear directions for clinical practice and future research.

Based on this framework we suggest that both gait assessment and cognitive assessment, with particular focus on executive function, should be integrated in fall risk screening. The results of these assessments should be interpreted and utilised in a multidisciplinary approach in a comprehensive fall-prevention program. Many studies have investigated isolated treatments in order to test causal pathways of fall risk factors in older adults with dementia. However, a comprehensive program to address all risk factors is lacking in the literature. We are proposing a multidisciplinary approach to close the gap based on this framework and merge those single treatments in a more comprehensive program. Strategies may include the prescription of walking aids appropriate to the individuals' physical as well as cognitive capacity, customised training programs targeting identified gait abnormalities in addition to conventional strength and balance training, non-pharmaceutical and pharmaceutical strategies to increase attention, and cognitive training to improve planning and cognitive flexibility/problem-solving skills with the aim of improving the individual's ability to mobilise safely during challenging circumstances. Finally, attempts to address decline in cognitive domains that contribute to risk-taking behaviour, such as poor judgment and inhibitory control, through behavioural change/modulation may be utilised to improve the ability to appraise risks when navigating and mobilising in the environment. Guided by the theoretical framework, incorporating these strategies in a comprehensive multidisciplinary fallprevention program may result in better outcomes in reducing fall risks in older adults with dementia.

\section{Acknowledgements}

The authors thank Dr. Kayla Maloney, data analysis officer from Digital Curation and Data Access Services at The University of Sydney for assisting diagram illustration, and Ms. Marcelle Segal and Ms. Tracey Stevens from Wolper Jewish Hospital, Sydney, for proofreading of the manuscript.

\section{Disclosure Statement}

The authors have no conflicts of interest to declare.

\section{Funding Sources}

Associate professor Lee-Fay Low is funded by an NHMRC boosting dementia leadership fellowship.

\section{Author Contributions}

All authors contributed to the study design. Based on discussions with all authors, Ms. Zhang drafted the manuscript, which all authors revised. All authors approved the final version submitted for publication. 
Zhang et al.: Review of Gait, Cognition, and Fall Risks

\section{References}

1 Taylor ME, Delbaere K, Lord SR, Mikolaizak AS, Brodaty H, Close JC. Neuropsychological, physical, and functional mobility measures associated with falls in cognitively impaired older adults. J Gerontol A Biol Sci Med Sci. 2014 Aug;69(8):987-95.

2 Close JC, Wesson J, Sherrington C, Hill KD, Kurrle S, Lord SR, et al. Can a tailored exercise and home hazard reduction program reduce the rate of falls in community dwelling older people with cognitive impairment: protocol paper for the i-FOCIS randomised controlled trial. BMC Geriatr. 2014 Aug;14(1):89.

3 Guo JL, Tsai YY, Liao JY, Tu HM, Huang CM. Interventions to reduce the number of falls among older adults with/without cognitive impairment: an exploratory meta-analysis. Int J Geriatr Psychiatry. 2014 Jul;29(7): 661-9.

4 Fernando E, Fraser M, Hendriksen J, Kim CH, Muir-Hunter SW. Risk factors associated with falls in older adults with dementia: a systematic review. Physiother Can. 2017;69(2):161-70.

5 Meyer C, Hill S, Dow B, Synnot A, Hill K. Translating falls prevention knowledge to community-dwelling older PLWD: a mixed-method systematic review. Gerontologist. 2015 Aug;55(4):560-74.

6 Härlein J, Dassen T, Halfens RJ, Heinze C. Fall risk factors in older people with dementia or cognitive impairment: a systematic review. J Adv Nurs. 2009 May;65(5):922-33.

7 Rudolph JL, Zanin NM, Jones RN, Marcantonio ER, Fong TG, Yang FM, et al. Hospitalization in communitydwelling persons with Alzheimer's disease: frequency and causes. J Am Geriatr Soc. 2010 Aug;58(8):1542-8.

8 Ungar A, Mussi C, Nicosia F, Ceccofiglio A, Bellelli G, Bo M, et al. The "syncope and dementia" study: a prospective, observational, multicenter study of elderly patients with dementia and episodes of "suspected" transient loss of consciousness. Aging Clin Exp Res. 2015 Dec;27(6):877-82.

9 Whitney J, Close JC, Jackson SH, Lord SR. Understanding risk of falls in people with cognitive impairment living in residential care. J Am Med Dir Assoc. 2012 Jul;13(6):535-40.

10 Epstein NU, Guo R, Farlow MR, Singh JP, Fisher M. Medication for Alzheimer's disease and associated fall hazard: a retrospective cohort study from the Alzheimer's Disease Neuroimaging Initiative. Drugs Aging. 2014 Feb;31(2):125-9.

11 Hartman YA, Karssemeijer EG, van Diepen LA, Olde Rikkert MG, Thijssen DH. Dementia patients are more sedentary and less physically active than age- and sex-matched cognitively healthy older adults. Dement Geriatr Cogn Disord. 2018;46(1-2):81-9.

12 McPherson S, Fairbanks L, Tiken S, Cummings JL, Back-Madruga C. Apathy and executive function in Alzheimer's disease. J Int Neuropsychol Soc. 2002 Mar;8(3):373-81.

13 Schwenk M, Hauer K, Zieschang T, Englert S, Mohler J, Najafi B. Sensor-derived physical activity parameters can predict future falls in people with dementia. Gerontology. 2014;60(6):483-92.

14 Lach HW, Harrison BE, Phongphanngam S. Falls and fall prevention in older adults with early-stage dementia: an integrative review. Res Gerontol Nurs. 2017 May;10(3):139-48.

15 Muir-Hunter SW, Montero-Odasso M. The attentional demands of ambulating with an assistive device in older adults with Alzheimer's disease. Gait Posture. 2017 May;54:202-8.

16 Bateni H, Maki BE. Assistive devices for balance and mobility: benefits, demands, and adverse consequences. Arch Phys Med Rehabil. 2005 Jan;86(1):134-45.

17 Deandrea S, Lucenteforte E, Bravi F, Foschi R, La Vecchia C, Negri E. Risk factors for falls in communitydwelling older people: a systematic review and meta-analysis. Epidemiology. 2010 Sep;21(5):658-68.

18 Sachdev PS, Blacker D, Blazer DG, Ganguli M, Jeste DV, Paulsen JS, et al. Classifying neurocognitive disorders: the DSM-5 approach. Nat Rev Neurol. 2014 Nov;10(11):634-42.

19 Yogev-Seligmann G, Hausdorff JM, Giladi N. The role of executive function and attention in gait. Mov Disord. 2008 Feb;23(3):329-42.

20 Oh-Park M, Salazar T. Geriatric gait and balance disorders. 2017 [cited 2018 Dec 6]. Available from: https:// now.aapmr.org/geriatric-gait-and-balance-disorders/

21 Holtzer R, Epstein N, Mahoney JR, Izzetoglu M, Blumen HM. Neuroimaging of mobility in aging: a targeted review. J Gerontol A Biol Sci Med Sci. 2014 Nov;69(11):1375-88.

22 Axer H, Axer M, Sauer H, Witte OW, Hagemann G. Falls and gait disorders in geriatric neurology. Clin Neurol Neurosurg. 2010 May;112(4):265-74.

23 Parihar R, Mahoney JR, Verghese J. Relationship of gait and cognition in the elderly. Curr Transl Geriatr Exp Gerontol Rep. 2013 Sep;2(3):167-73.

24 Nutt JG, Marsden CD, Thompson PD. Human walking and higher-level gait disorders, particularly in the elderly. Neurology. 1993 Feb;43(2):268-79.

25 Martin MP, O’Neill D. Vascular higher-level gait disorders - a step in the right direction? Lancet. 2004 Jan; 363(9402):8.

26 Nutt JG. Classification of gait and balance disorders. Adv Neurol. 2001;87:135-41.

27 Domellöf ME, Elgh E, Forsgren L. The relation between cognition and motor dysfunction in drug-naive newly diagnosed patients with Parkinson's disease. Mov Disord. 2011 Oct;26(12):2183-9.

28 Cohen JA, Verghese J, Zwerling JL. Cognition and gait in older people. Maturitas. 2016 Nov; 93:73-7.

29 Bahureksa L, Najafi B, Saleh A, Sabbagh M, Coon D, Mohler MJ, et al. The impact of mild cognitive impairment on gait and balance: a systematic review and meta-analysis of studies using instrumented assessment. Gerontology. 2017;63(1):67-83. 
30 Beauchet O, Annweiler C, Callisaya ML, De Cock AM, Helbostad JL, Kressig RW, et al. Poor gait performance and prediction of dementia: results from a meta-analysis. J Am Med Dir Assoc. 2016 Jun;17(6):482-90.

31 Montero-Odasso M, Speechley M. Falls in cognitively impaired older adults: implications for risk assessment and prevention. J Am Geriatr Soc. 2018 Feb;66(2):367-75.

32 Rosso AL, Studenski SA, Chen WG, Aizenstein HJ, Alexander NB, Bennett DA, et al. Aging, the central nervous system, and mobility. J Gerontol A Biol Sci Med Sci. 2013 Nov;68(11):1379-86.

33 Bonelli RM, Cummings JL. Frontal-subcortical circuitry and behavior. Dialogues Clin Neurosci. 2007;9(2): 141-51.

34 Rosano C, Brach J, Studenski S, Longstreth WT Jr, Newman AB. Gait variability is associated with subclinical brain vascular abnormalities in high-functioning older adults. Neuroepidemiology. 2007;29(3-4):193-200.

35 Rosano C, Studenski SA, Aizenstein HJ, Boudreau RM, Longstreth WT Jr, Newman AB. Slower gait, slower information processing and smaller prefrontal area in older adults. Age Ageing. 2012 Jan;41(1):58-64.

36 Amboni M, Barone P, Hausdorff JM. Cognitive contributions to gait and falls: evidence and implications. Mov Disord. 2013 Sep;28(11):1520-33.

37 Wennberg AM, Savica R, Mielke MM. Association between various brain pathologies and gait disturbance. Dement Geriatr Cogn Disord. 2017;43(3-4):128-43.

38 Holtzer R, Mahoney JR, Izzetoglu M, Wang C, England S, Verghese J. Online fronto-cortical control of simple and attention-demanding locomotion in humans. Neuroimage. 2015 May;112:152-9.

39 Mahoney JR, Holtzer R, Izzetoglu M, Zemon V, Verghese J, Allali G. The role of prefrontal cortex during postural control in Parkinsonian syndromes a functional near-infrared spectroscopy study. Brain Res. 2016 Feb;1633: 126-38.

40 Martin KL, Blizzard L, Wood AG, Srikanth V, Thomson R, Sanders LM, et al. Cognitive function, gait, and gait variability in older people: a population-based study. J Gerontol A Biol Sci Med Sci. 2013 Jun;68(6):726-32.

41 Seidler RD, Bernard JA, Burutolu TB, Fling BW, Gordon MT, Gwin JT, et al. Motor control and aging: links to age-related brain structural, functional, and biochemical effects. Neurosci Biobehav Rev. 2010 Apr;34(5): 721-33.

42 Li KZ, Bherer L, Mirelman A, Maidan I, Hausdorff JM. Cognitive involvement in balance, gait and dual-tasking in aging: a focused review from a neuroscience of aging perspective. Front Neurol. 2018 Oct;9:913.

43 Liu-Ambrose T, Nagamatsu LS, Hsu CL, Bolandzadeh N. Emerging concept: 'central benefit model' of exercise in falls prevention. Br J Sports Med. 2013 Jan;47(2):115-7.

44 van Ooijen MW, Roerdink M, Trekop M, Janssen TW, Beek PJ. The efficacy of treadmill training with and without projected visual context for improving walking ability and reducing fall incidence and fear of falling in older adults with fall-related hip fracture: a randomized controlled trial. BMC Geriatr. 2016 Dec;16(1):215.

45 Zhang W, Low LF, Gwynn JD, Clemson L. Interventions to improve gait in older adults with cognitive impairment: A systematic review. J Am Geriatr Soc. 2019 Feb;67(2):381-91.

46 Beauchet O, Launay CP, Allali G, Watfa G, Gallouj K, Herrmann FR, et al. Anti-dementia drugs and changes in gait: a pre-post quasi-experimental pilot study. BMC Neurol. 2013 Nov;13(1):184.

47 Beauchet $\mathrm{O}$, Launay CP, Allali G, Annweiler C. Changes in gait variability with anti-dementia drugs: a systematic review and meta-analysis. CNS Drugs. 2014 Jun;28(6):513-8.

48 Snijders AH, van de Warrenburg BP, Giladi N, Bloem BR. Neurological gait disorders in elderly people: clinical approach and classification. Lancet Neurol. 2007 Jan;6(1):63-74.

49 van der Wardt V, Logan P, Hood V, Booth V, Masud T, Harwood R. The association of specific executive functions and falls risk in people with mild cognitive impairment and early-stage dementia. Dement Geriatr Cogn Disord. 2015;40(3-4):178-85.

50 Ambrose AF, Paul G, Hausdorff JM. Risk factors for falls among older adults: a review of the literature. Maturitas. 2013 May; 75(1):51-61.

51 Stuss DT, Alexander MP. Executive functions and the frontal lobes: a conceptual view. Psychol Res. 2000;63(34):289-98.

52 Segev-Jacubovski O, Herman T, Yogev-Seligmann G, Mirelman A, Giladi N, Hausdorff JM. The interplay between gait, falls and cognition: can cognitive therapy reduce fall risk? Expert Rev Neurother. 2011 Jul;11(7):105775.

53 Mirelman A, Herman T, Brozgol M, Dorfman M, Sprecher E, Schweiger A, et al. Executive function and falls in older adults: new findings from a five-year prospective study link fall risk to cognition. PLoS One. 2012; 7(6):e40297.

54 Haworth JM. Gait, aging and dementia. Rev Clin Gerontol. 2008;18(1):39-52.

55 Springer S, Giladi N, Peretz C, Yogev G, Simon ES, Hausdorff JM. Dual-tasking effects on gait variability: the role of aging, falls, and executive function. Mov Disord. 2006 Jul;21(7):950-7.

56 Lezak MD, Howieson DB, Bigler ED, Tranel D. Neuropsychological assessment. 5th ed. New York, NY, US: Oxford University Press; 2012.

57 Muir SW, Berg K, Chesworth B, Klar N, Speechley M. Balance impairment as a risk factor for falls in communitydwelling older adults who are high functioning: a prospective study. Phys Ther. 2010 Mar;90(3):338-47.

58 Festa EK, Insler RZ, Salmon DP, Paxton J, Hamilton JM, Heindel WC. Neocortical disconnectivity disrupts sensory integration in Alzheimer's disease. Neuropsychology. 2005 Nov;19(6):728-38.

59 Camarda R, Camarda C, Monastero R, Grimaldi S, Camarda LK, Pipia C, et al. Movements execution in amnestic mild cognitive impairment and Alzheimer's disease. Behav Neurol. 2007;18(3):135-42. 
60 Pieruccini-Faria F, Jones JA, Almeida QJ. Motor planning in Parkinson's disease patients experiencing freezing of gait: the influence of cognitive load when approaching obstacles. Brain Cogn. 2014 Jun;87:76-85.

61 Hobert MA, Meyer SI, Hasmann SE, Metzger FG, Suenkel U, Eschweiler GW, et al. Gait is associated with cognitive flexibility: a dual-tasking study in healthy older people. Front Aging Neurosci. 2017 May;9(154):154.

62 Kearney FC, Harwood RH, Gladman JR, Lincoln N, Masud T. The relationship between executive function and falls and gait abnormalities in older adults: a systematic review. Dement Geriatr Cogn Disord. 2013;36(1-2): 20-35.

63 Lowry KA, Sebastian K, Perera S, Van Swearingen J, Smiley-Oyen AL. Age-related differences in locomotor strategies during adaptive walking. J Mot Behav. 2017 Jul-Aug; 49(4):435-40.

64 Liu-Ambrose T, Ahamed Y, Graf P, Feldman F, Robinovitch SN. Older fallers with poor working memory overestimate their postural limits. Arch Phys Med Rehabil. 2008 Jul;89(7):1335-40.

65 Diamond A. Executive functions. Annu Rev Psychol. 2013;64(1):135-68.

66 Uemura K, Hasegawa T, Tougou H, Shuhei T, Uchiyama Y. Analysis of choice stepping with visual interference can detect prolonged postural preparation in older adults with mild cognitive impairment at high risk of falling. Dement Geriatr Cogn Disord. 2015;40(1-2):13-21. 